\title{
Clinical exome sequencing identifies two novel mutations of the SCN1A and SCN2A genes in Moroccan patients with epilepsy: a case series
}

\author{
Maryem Sahli ${ }^{1,2,4^{*}}$, Abdelali Zrhidri ${ }^{1,2}$, Siham Chafai Elaloui ${ }^{1}$, Wiam Smaili ${ }^{2}$, Jaber Lyahyai ${ }^{1}$,
} Fatima Zohra Oudghiri ${ }^{3}$ and Abdelaziz Sefiani ${ }^{1,2}$

\begin{abstract}
Background: Epilepsy is the most common neurological disorder that causes spontaneous, unprovoked, and recurrent seizures. Epilepsy is clinically and genetically heterogeneous with various modes of inheritance. The complexity of epilepsy presents a challenge and identification of the causal genetic mutation allows diagnosis, genetic counseling, predicting prognosis, and, in some cases, treatment decisions. Clinical exome sequencing is actually becoming a powerful approach for molecular diagnosis of heterogeneous neurological disorders in clinical practice.

Case presentation: We report our observations of three unrelated Moroccan patients referred to our genetics department for molecular diagnosis of epilepsy: a 4-year-old Moroccan boy, a 3-year-old Moroccan girl, and a 7-year-old Moroccan boy. Due to the heterogeneity and complexity of epilepsy, we performed clinical exome sequencing followed by targeted analysis of 936 epilepsy genes. A total of three mutations were identified in known epilepsy genes (SCN1A, SCN2A). By clinical exome sequencing, we identified two novel mutations: c.4973C >A (p.Thr1658Lys) in SCN1A gene and c.1283A>G (p.Tyr428Cys) in the SCN2A gene, whereas the third mutation c.3295G>T (p.Glu1099*) was already described in patients with Dravet syndrome.
\end{abstract}

Conclusion: This study demonstrates that clinical exome sequencing is an effective diagnosis tool to investigate this group of diseases with huge diversity and defends its use in clinical routine.

Keywords: Epilepsy, Clinical exome sequencing, SCN1A, SCN2A, Novel mutation, Moroccan

\section{Background}

Epilepsy or "seizures disorders" is the most common neurological disorder. The prevalence of epilepsy is 6.38 per 1000 individuals, and approximately 50 million people are affected worldwide [1,2]. Epilepsy includes a large neurological condition characterized by spontaneous, unprovoked, recurrent, and unpredictable seizures

\footnotetext{
* Correspondence: sahlimaryem1990@gmail.com; sahlimaryem1990@gmail.fr ${ }^{1}$ Centre de Recherche en Génomique des Pathologies Humaines (GENOPATH), Faculté de Médecine et de Pharmacie, Mohammed V University of Rabat, 10100 Rabat, Morocco

²Département de génétique médicale, Institut National d'Hygiène, BP 769 Agdal, 10090 Rabat, Morocco

Full list of author information is available at the end of the article
}

[3]. Epilepsy can be isolated or a clinical feature of a syndrome with additional signs [4].

The causes of epilepsy are multiple. The causes involve genetic factors in $70-80 \%$ of cases of epilepsy, which constitute the main factor in idiopathic epilepsies [5]. On the other hand, a large proportion of cases of epilepsy are caused by acquired conditions such as cerebral trauma, cerebral tumor, cerebral infection, cerebrovascular disorders, and inborn errors of metabolism [6]. Because of the phenotypic and genetic heterogeneity of this group of diseases, we opted for clinical exome sequencing (CES) as a diagnosis approach to allow a genetic diagnosis for our patients.

(c) The Author(s). 2019 Open Access This article is distributed under the terms of the Creative Commons Attribution 4.0 International License (http://creativecommons.org/licenses/by/4.0/), which permits unrestricted use, distribution, and 
We report here the observations of three unrelated patients referred to our Department of Medical Genetics for epilepsy of undetermined origin, for genetic study. As a result, we identified causal mutations in the three analyzed patients, including two novel mutations.

\section{Case presentation}

\section{Patients}

Three patients were referred to our Department of Medical Genetics in Rabat for epilepsy without previous clinical diagnosis. Informed parental consent for genetic testing was obtained for all participants of this study. Clinical diagnoses and all detected variants are summarized in Table 1.

\section{Patient 1}

Our first patient is a 4-year-old Moroccan boy, non-consanguineous, the last child of three siblings, referred for Dravet syndrome. His history showed normal psychomotor development until 6 months of age. Daily generalized seizures started at 6 months of life and were treated with sodium valproate, clobazam, and carbamazepine but without any response. At clinical examination, growth parameters were within normal limits with no facial dysmorphia. Magnetic resonance imaging (MRI) was normal; however, an electroencephalogram (EEG) showed an extended EEG and isolated points were seen over the frontocentral region.

\section{Patient 2}

Our second patient is a 3-year-old Moroccan girl referred to our Department of Medical Genetics for uncontrolled seizures. She was born at term after an uneventful pregnancy; her birth weight was $3.2 \mathrm{~kg}$. She was the only child of a non-consanguineous couple. At the age of 5 months, she developed generalized seizure following a fever. She was treated with sodium valproate and clobazam but without any response. At clinical examination, she had stature and weight delay at < third percentile, microcephaly at - 2SD, with no facial dysmorphia. MRI scans showed normal results.

\section{Patient 3}

Our third patient is a 7-year-old Moroccan boy who presented to our department with idiopathic epilepsy and developmental regression. His history showed normal psychomotor development until 21 months of life. Treatment with sodium valproate and clobazam was initiated with poor seizure control. At physical examination, he had stature at $<$ third percentile, microcephaly at -2 SD, with no facial dysmorphia. An MRI was normal but EEG showed generalized tonic seizures.

\section{CES and data analysis}

We tested three unrelated patients with epilepsy of undetermined origin. Due to the phenotypic and genetic heterogeneity of epilepsy and no hot spots mutation, we used CES as a diagnosis strategy for individuals with this clinical entity.

The CES was performed using the SOPHiA ${ }^{\text {tw }}$ Genetics CESV1 kit on NextSeq 500 (Illumina). Raw data were analyzed, annotated, and filtering steps were performed using SOPHiA $^{\text {tix }}$ DDM (SOPHiA Genetics ${ }^{\circ}$ ). Variants with allele frequencies above 2\% in Exome Sequencing Project (ESP) 6500 , and variants not predicted to be deleterious were excluded. CES results were further validated using Sanger sequencing by designing the primers of the identified

Table 1 Clinical and genetic findings of the three Moroccan patients with epilepsy

\begin{tabular}{|c|c|c|c|}
\hline Number & Patient 1 (P1) & Patient 2 (P2) & Patient 3 (P3) \\
\hline Age & 4 years & 3 years & 7 years \\
\hline Age at onset & 6 months & 5 months & 21 months \\
\hline Sex & M & $\mathrm{F}$ & M \\
\hline Consanguineous & No & No & No \\
\hline Psychomotor development retardation & Yes & Yes & Yes \\
\hline Interictal EEG & $\begin{array}{l}\text { Extended EEG isolated points are seen } \\
\text { over the frontocentral region }\end{array}$ & NA & Tonic seizures \\
\hline Cerebral MRI & $\mathrm{N}$ & $\mathrm{N}$ & $\mathrm{N}$ \\
\hline Drugs resistance & Yes & Yes & No \\
\hline Gene/Transcript & SCN1A/NM_001202435 & SCN1A/NM_001202435 & SCN2ANM_021007.2 \\
\hline Nucleotide change & c. $4973 C>A$ & c.3295G >T & c. $1283 A>G$ \\
\hline Amino acid change & p.Thr1658Lys & p.Glu1099* & p.Tyr428Cys \\
\hline Inheritance & $A D$ & $A D$ & $A D$ \\
\hline Origin of variant & De novo & De novo & De novo \\
\hline
\end{tabular}

$A D$ autosomal dominant, EEG electroencephalogram, $F$ female, $M$ male, $M R I$ magnetic resonance imaging, $N$ normal, $N A$ not available 
mutations. Amplification products were electrophoresed on $1 \%$ agarose gels. Sanger sequencing was done with dye terminator chemistry (ABI Prism ${ }^{\circ}$ BigDye v3.1) and run on automated sequencer Applied Biosystems Prism 3130 DNA Analyzer. Obtained sequences were aligned to the reference genome (GRCh37/hg19) using DNA Variant analysis software (Mutation Surveyor ${ }^{\natural}$ software).

The established variants were cross-checked with the 1000 Genomes Project database (http://www.1000genomes.org/), with the Exome Variant Server (http://evs.gs. washington.edu/EVS/), Human Gene Mutation Database (HGMD; http://www.biobase-international.com/product/ hgmd), and with the "ClinVar" database (http://www.ncbi. nlm.nih.gov/clinvar/).

\section{Discussion}

Epilepsy is the most common neurological disorder; it is characterized by a broad clinical and genetic spectrum. The clinical heterogeneity and molecular complexity of this group of diseases pose a real challenge to clinicians for diagnosis and treatment. CES is actually becoming a powerful approach for molecular diagnosis of heterogeneous neurological disorders such as epilepsy in clinical practice. Therefore, it is considered a new approach of molecular diagnosis to identify mutations in patients with rare and heterogeneous genetic diseases facing a large genetic differential diagnosis [7].

Due to the phenotypic and genetic heterogeneity of neurological disorders, especially epilepsy, many teams use gene panels as a diagnosis approach. However, given the large number of genetic diseases with epilepsy as a symptom and given that gene panels developed by each laboratory differ greatly, CES is becoming an effective common molecular diagnostic tool for individuals with a heterogeneous phenotype. In addition, epilepsy gene panels are multiple and so far there are no next-generation sequencing (NGS) guidelines for patients with epilepsy [8]. Currently, there is a huge diversity in the number and composition of genes of the different epilepsy panels commercially available [9-12]. Gene panels are also expensive and associated with a low diagnosis yield [13].

CES analysis is associated with a decrease in cost and a high diagnosis yield in comparison to other genetic tests used currently in diagnosis, including sequencing the whole gene and gene panels, and could be used in patients with epilepsy to identify mutations and allow a definitive diagnosis, to adapt antiepileptic drug, to predict the prognosis, and to provide genetic counseling to families.

As a department of medical genetics providing genetic services and testing for various diseases in a country with lower income, molecular diagnosis strategies by NGS or single gene sequencing with Sanger of heterogeneous diseases like epilepsy may represent a real challenge. It is obvious that we design an appropriate panel of each disease and that would be highly efficient. However, the very limited number of cases of epilepsy among the various genetic diseases from our consultation and the significant cost of dedicated panels bring us to consider a customized multigenes panel approach. This strategy of diagnosis with CES is better adapted to our diversified requests of molecular tests.

In this study, we performed CES in three unrelated patients with epilepsy of undetermined origin. We detected causal mutations in all cases; the three mutations were found in two genes, SCN1A and SCN2A, which encode the brain sodium channel. Two mutations were novel findings, highlighting the value of CES to detect de novo mutations especially when applied to heterogeneous disorders such as epilepsy.

In our first patient, a heterozygous mutation was identified: c.4973C >A (p.Thr1658Lys) of the SCN1A gene (NM 001202435) (see Table 1). This variant has never been reported in public databases; however, the change of amino acid threonine in position 1658 to another amino acid has been reported several times in patients with Dravet syndrome. The p.Thr1658Lys of the SCN1A gene was predicted to be probably damaging by a bioinformatic analysis using PolyPhen and SIFT tools. PolyPhen predicted it as possibly damaging, while SIFT predicted it as deleterious or disease causing.

Our second patient had a heterozygous mutation c.3295G>T (p.Glu1099*) of the SCN1A gene (see Table 1), this mutation was already identified in patients showing Dravet syndrome [14].

For our third patient we detected a single heterozygous mutation in coding exon 10: c.1283A $>$ G (p.Tyr428Cys) of the SCN2A gene (NM_021007.2) (see Table 1). The mutation in the $S C N 2 A$ gene in our patient is considered pathogenic because it is a de novo mutation and occurred in an evolutionary conserved amino acid at this position of the protein. In addition, this variant was absent from the control population (Exome Aggregation Consortium, Cambridge, MA). The c. $1283 \mathrm{~A}>\mathrm{G}$ of the $S C N 2 A$ gene is a novel mutation, and it had never been reported in the literature to the best of our knowledge.

\section{Conclusion}

In conclusion, these observations showed that CES is a powerful tool to detect de novo mutations, especially when applied to heterogeneous neurologic phenotypes, such as epilepsy, facing a broad genetic differential diagnosis and defend its application in clinical practice.

\section{Abbreviations}

CES: Clinical exome sequencing; EEG: Electroencephalogram; MRI: Magnetic resonance imaging; NGS: Next-generation sequencing 


\section{Acknowledgements}

We thank the patients and their families.

\section{Authors' contributions}

MS carried out the clinical diagnosis, literature search, and manuscript preparation. AZ and JL analyzed the patients' data and helped in writing the manuscript. SE carried out the clinical diagnosis and contributed in writing the manuscript. FO and AS participated in the design of the study and manuscript review. All authors read and approved the final manuscript.

\section{Availability of data and materials}

Available on request.

\section{Ethics approval and consent to participate}

Signed informed consents from patients' parents were obtained to perform DNA analysis. This study was approved by the ethics committee of the National Institute of Health in Rabat, Morocco.

\section{Consent for publication}

Written informed consent was obtained from the patients' legal guardians for publication of this case report and any accompanying images. A copy of the written consents is available for review by the Editor-in-Chief of this journal.

\section{Competing interests}

The authors declare that they have no competing interests.

\section{Author details}

${ }^{1}$ Centre de Recherche en Génomique des Pathologies Humaines (GENOPATH), Faculté de Médecine et de Pharmacie, Mohammed V University of Rabat, 10100 Rabat, Morocco. ${ }^{2}$ Département de génétique médicale, Institut National d'Hygiène, BP 769 Agdal, 10090 Rabat, Morocco. ${ }^{3}$ Unité de Neuropédiatrie, Hôpital d'Enfants de Rabat, Rabat, Morocco. ${ }^{4}$ Département de Génétique Médicale, Institut National d'Hygiène, 7Avenue Ibn Batouta, B.P. 769, 11400 Rabat, Morocco.

Received: 29 March 2019 Accepted: 17 July 2019

Published online: 23 August 2019

\section{References}

1. Fiest KM, Sauro KM, Wiebe S, et al. Prevalence and incidence of epilepsy: A systematic review and meta-analysis of international studies. Neurology. 2017; 88(3):296-303.

2. Santhosh NS, Sinha S, Satishchandra P. Epilepsy: Indian perspective. Ann Indian Acad Neurol. 2014;17(Suppl 1):S3-S11.

3. Fisher RS, van Emde Boas W, Blume W, et al. Epileptic seizures and epilepsy: definitions proposed by the International League Against Epilepsy (ILAE) and the International Bureau for Epilepsy (IBE). Epilepsia. 2005:46(4):470-2.

4. Matricardi S, Canafoglia L, Ardissone A, et al. Epileptic phenotypes in children with early-onset mitochondrial diseases. Acta Neurol Scand. 2019; https://doi.org/10.1111/ane.13130.

5. Myers $\mathrm{CT}$, Mefford $\mathrm{HC}$. Advancing epilepsy genetics in the genomic era. Genome Med. 2015;7:91.

6. Liu S, Yu W, Lu Y. The causes of new-onset epilepsy and seizures in the elderly. Neuropsychiatr Dis Treat. 2016;12:1425-34.

7. Fogel BL, Satya-Murti S, Cohen BH. Clinical exome sequencing in neurologic disease. Neurol Clin Pract. 2016;6(2):164-76.

8. Tumiene B, Maver A, Writzl K, et al. Diagnostic exome sequencing of syndromic epilepsy patients in clinical practice. Clin Genet. 2018;93(5): 1057-62.

9. Epi4K Consortium; Epilepsy Phenome/Genome Project, Allen AS, et al. De novo mutations in epileptic encephalopathies. Nature. 2013; 501(7466):217-21.

10. Kodera H, Kato M, Nord AS, et al. Targeted capture and sequencing for detection of mutations causing early onset epileptic encephalopathy. Epilepsia. 2013;54(7):1262-9.

11. Allen NM, Conroy J, Shahwan A, et al. Unexplained early onset epileptic encephalopathy: Exome screening and phenotype expansion. Epilepsia. 2016;57(1):e12-7.

12. Xue Y, Ankala A, Wilcox WR, Hegde MR. Solving the molecular diagnostic testing conundrum for Mendelian disorders in the era of next-generation sequencing: single-gene, gene panel, or exome/ genome sequencing. Genet Med. 2015;17(6):444-51.

13. Usluer S, Salar S, Arslan M, et al. SCN1A gene sequencing in 46 Turkish epilepsy patients disclosed 12 novel mutations. Seizure. 2016;39:34-43.

14. Mancardi MM, Striano $P$, Gennaro E, et al. Familial occurrence of febrile seizures and epilepsy in severe myoclonic epilepsy of infancy (SMEI) patients with SCN1A mutations. Epilepsia. 2006;47(10):1629-35.

\section{Publisher's Note}

Springer Nature remains neutral with regard to jurisdictional claims in published maps and institutional affiliations.
Ready to submit your research? Choose BMC and benefit from:

- fast, convenient online submission

- thorough peer review by experienced researchers in your field

- rapid publication on acceptance

- support for research data, including large and complex data types

- gold Open Access which fosters wider collaboration and increased citations

- maximum visibility for your research: over $100 \mathrm{M}$ website views per year

At $\mathrm{BMC}$, research is always in progress.

Learn more biomedcentral.com/submissions 